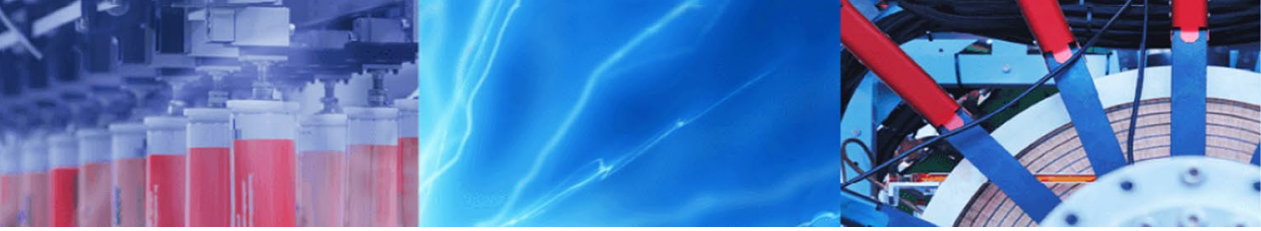

Research Article

\title{
Simultaneous determination of anthelmintic drugs by capillary electrophoresis using cyclodextrins as buffer additives
}

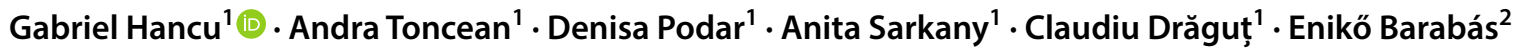

(c) Springer Nature Switzerland AG 2019

\begin{abstract}
A generic capillary zone electrophoresis with diode array detection was developed for the simultaneous determination of five anthelmintic drugs (albendazole, fenbendazole, flubendazole, mebendazole, levamisole) used in human and veterinary medicine. The separation of structurally related benzimidazole derivatives was resolved by addition in the background electrolyte of randomly methylated $\beta$-cyclodextrin (RAMEB). The optimization of the analytical conditions was carried out using a "one factor at a time" tehnique. Optimized conditions were found to be $50 \mathrm{mM}$ phosphate buffer at $\mathrm{pH} 1.9,5 \mathrm{mM}$ RAMEB as buffer additive, applied voltage $+25 \mathrm{kV}$, system temperature $20^{\circ} \mathrm{C}$, injection parameters $50 \mathrm{mbar} / 1 \mathrm{~s}$. Good analytical performance with regards to linearity, reproducibility, accuracy and robustness was achieved. The method was applied for the determination of analytes from pharmaceutical preparations.
\end{abstract}

Keywords Anthelmintic · Benzimidazole drugs · Capillary electrophoresis · Cyclodextrins

\section{Introduction}

Helminthiasis is among the most common infections in man, affecting a large proportion of the world's population; these infections can affect large populations in endemic areas with major economic and social consequences [1].

Benzimidazoles are a broad-spectrum class of anthelmintic drugs which are used in both humans and animals in the treatment of roundworm and tapeworm infestations. Since the introduction in therapy of the first benzimidazole derivative, thiabendazole, nearly 50 years ago, many benzimidazoles and pro-benzimidazoles with increased efficacy and spectra have been synthetized and used in therapy [2].

In this study four benzimidazole derivatives: albendazole (ABZ) (methyl N-(6-propylsulfanyl-1H-benzimidazol-2-yl)carbamate), fenbendazole (FBZ) (methyl $\mathrm{N}$-(6-phenylsulfanyl-1H-benzimidazol-2-yl)carbamate), flubendazole (FLZ) (methyl N-[6-(4-fluorobenzoyl)-1H-benzimidazol-2-yl]carbamate), mebendazole (MBZ) (methyl $\mathrm{N}$-(6-benzoyl-1H-benzimidazol-2-yl)carbamate) and one imidazothiazole derivative: levamisole (LEV) ((6S)-6-phenyl-2,3,5,6-tetrahydroimidazo[2,1-b] [1, 3] thiazole) were analysed by capillary electrophoresis. The chemical structures of the studied analytes are presented in Fig. 1.

Nowadays capillary electrophoresis (CE) is a powerful separation technique in pharmaceutical analysis being used in research, development, quality control or stability studies of pharmaceutical substances [3]. Comparing it with the more frequently used high performance liquid chromatography (HPLC), CE has the advantages of rapid method development, higher resolution capacity, different mechanism for separation selectivity, low consumption of analytes and reagents and consequently low operational costs [4].

In recent years several CE methods for the simultaneous determination of benzimidazole from different matrices

Gabriel Hancu, gabriel.hancu@umftgm.ro| 'Department of Pharmaceutical Chemistry, Faculty of Pharmacy, University of Medicine, Pharmacy, Science and Technology from Tîrgu Mureș, Tîrgu Mureș, Romania. ${ }^{2}$ Department of Cell Biology and Microbiology, Faculty of Pharmacy, University of Medicine, Pharmacy, Science and Technology from Tîrgu Mureș, Tîrgu Mureș, Romania. 
Fig. 1 Chemical structures of the studied anthelmintic drugs<smiles>COC(=O)Nc1nc2cc(C(=O)c3ccccc3)ccc2[nH]1</smiles>

Mebendazole (MBZ)<smiles>COC(=O)Nc1nc2cc(C(=O)c3ccc(F)cc3)ccc2[nH]1</smiles>

Flubendazole (FLZ)<smiles>CCCSc1ccc2[nH]c(NC(=O)OC)nc2c1</smiles>

Albendazole (ABZ)<smiles>COC(=O)Nc1nc2cc(Sc3ccccc3)ccc2[nH]1</smiles>

Fenbendazole (FBZ)

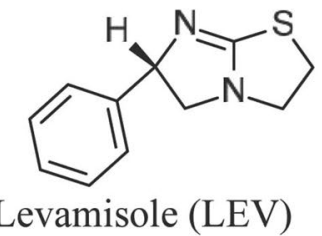

has been published. Benzimidazole residues in swine tissue samples were determined by magnetic solid phase extraction (MSPE) with field-amplified sample stacking technique (FASS) using a capillary zone electrophoresis (CZE) [5]. CE-MS methods were proposed for the determination of benzimidazole derivatives in egg [6] and meat [7] samples. Capillary electrochromatography (CEC) was used for the analysis of benzimidazoles in water residues [8]. Micellar electrokinetic chromatography (MEKC) was used for the simultaneous determination of three benzimidazole fungicides [9]. A high-performance CE method for determination of five benzimidazoles in animal feeds was also developed [10].

CE methods were also developed for the individual determination of certain benzimidazole derivatives. A nonaqueous capillary electrophoretic method (NACE) was developed for the determination of plasma levels of albendazole and its metabolites (albendazole sulfoxide, albendazole sulfone) [11]. Chiral CE was used for the determination of albendazole sulfoxide enantiomers in cerebrospinal fluid [12] and human plasma [13].

Usually analytical methods for determination of benzimidazoles are developed individually; this approach to the analysis is chosen because benzimidazoles are not used in combination with other benzimidazole during anthelminthic therapy. Levamisole however is sometimes used in combination with mebendazole in order to obtain a more effective broad-spectrum anthelmintic treatment. A NACE method was employed for the simultaneous determination of mebendazole and levamisole hydrochloride in fixed dosed combinations [14].

The development of a rapid analytical method that will not be limited to the analysis of only one benzimidazole derivative could be a useful approach. Therefore, the purpose of this study was the development of a generic universal $C E$ method for the determination of $A B Z, F B Z$, FLZ, MBZ and LEV in a single run without the need for development of a separate and distinct method for each substance.

\section{Materials and method}

\subsection{Chemicals and reagents}

We used the following substances of pharmaceutical grade: albendazole-ABZ (Tonira Pharma Limited, India), fenbendazole-FBZ (Transchem Limited, India), flubendazole-FLZ (Transchem Limited, India), mebendazoleMBZ (Tonira Pharma Limited, India), levamisole-LEV (Shandong Qilu King Phar, China).

The following reagents of analytical grade were used: phosphoric acid (85\%) (Chimopar, Romania), methanol, sodium hydroxide (Lach-Ner, Czech Republic), sodium dihydrogen phosphate, disodium hydrogen phosphate (Merck, Germany). Deionized water was purified by a 
Milli-Q system (Millipore, USA) and was used throughout for the preparation of solutions.

For the separation of the studied analytes, an addition of cyclodextrins (CDs) in the background electrolyte was used: native $C D s(a-C D, \beta-C D, \gamma-C D)$ and derivatized CDs (hydroxypropyl- $\beta-C D-H P-\beta-C D$, randomly methylated $\beta-C D$ - RAMEB, heptakis(2,3,6-tri-O-methyl) $\beta-C D$-TRIMEB).

To determine the concentration of $A B Z$ and $M B Z$ from pharmaceutical products, we used the Duador film-coated tablets (Gedeon Richter, Romania) containing 200 mg ABZ and Vermox tablets (Janssen, Belgium) containing $100 \mathrm{mg}$ MBZ.

\subsection{Instrumentation}

Determinations were conducted on an Agilent CE 1600 system (Agilent Technologies, Germany), equipped with a photo diode array detection. Data acquisition was performed using ChemStation software (Agilent Technologies, Germany). Uncoated fused-silica capillaries of $48.5 \mathrm{~cm}$ length (40 cm effective length) $\times 50 \mu \mathrm{m}$ I.D. (Agilent Technologies, Germany) were used. Buffer $\mathrm{pH}$ was determined using a Terminal 740 pH-meter (Inolab, Germany).

\subsection{Standard solutions preparation}

Due to the fact that benzimidazole derivatives could not be dissolved in methanol, stock solutions were prepared at concentrations of $1 \mathrm{mg} / \mathrm{mL}$ in a mixture of formic acid:methanol (1:4) while LEV stock solution was prepared directly in methanol in the same concentration. Working solutions were prepared by diluting the stock solution with methanol to the appropriate concentration. All stock solutions were stored in a refrigerator at $4{ }^{\circ} \mathrm{C}$ and were found to be stable for at least 2 weeks.

\subsection{Pharmaceutical sample preparations}

Ten tablets from each sample were weighed, triturated and homogenized in a mortar. An amount of powder equivalent to the average weight of one tablet was individually transferred and extracted in $10 \mathrm{~mL}$ mixture of formic acid:methanol (1:4), sonicated for $5 \mathrm{~min}$ and then diluted to $100 \mathrm{~mL}$ with methanol. The solutions were then diluted with methanol to the appropriate concentrations to fit the calibration curve plotted in the stock solution linearity analysis and filtered through a $0.45 \mu \mathrm{m}$ regenerated cellulose membrane filter before being subjected to the CE analysis.

\subsection{Electrophoretic conditions}

The new capillaries were conditioned by flushing for 30 min with $1 \mathrm{M} \mathrm{NaOH}, 15 \mathrm{~min}$ with $0.1 \mathrm{M} \mathrm{NaOH}$ and 15 min with water. Between runs, the capillaries were preconditioned for 2 min with $0.1 \mathrm{M} \mathrm{NaOH}$ and 3 min with the BGE prior to each subsequent run.

The CE system was operated under the following initial conditions: voltage, $25 \mathrm{kV}$, capillary temperature, $25^{\circ} \mathrm{C}$, hydrodynamic injection, $50 \mathrm{mbar}$ for $1 \mathrm{~s}$, detection wavelength, $210 \mathrm{~nm}$.

Buffer solutions were prepared by dissolving the appropriate amounts of reagents in water; the $\mathrm{pH}$ of the buffer solutions was adjusted with $0.1 \mathrm{~N}$ sodium hydroxide. All standard, sample solutions and BGE were filtered through a $0.45 \mu \mathrm{m}$ regenerated cellulose membrane filter.

\section{Results and discussion}

\subsection{Preliminary analysis}

Benzimidazole and imidazothiazole derivatives are slightly basic; the basic properties resulting from the nitrogen of the imidazole ring, which can accept protons. In acidic solution, benzimidazoles are protonated to form benzimidazolium ion [15].

The $\mathrm{pK}_{\mathrm{a}}$ value (ABZ-6.9; FBZ-7.4; FLZ-7.1; MBZ-6.6; LEV-6.9) of the drugs serves as a guideline that shows the extent of ionization at different $\mathrm{pH}$ levels [16]. In our case all substances have almost similar pKa values and will be ionized at the imidazole ring, in an acidic background electrolyte (BGE). We sought to establish optimal electrophoretic conditions for the separation by carrying out a series of preliminary experiments using different phosphate buffer systems over a $\mathrm{pH}$ range of 1.9 to 7.0 , at four pH levels $(1.9,3.5,5.0,7.0)$.

Choosing a suitable buffer system and $\mathrm{pH}$ is essential in the separation process since it will implicitly generate ionization and influence the electrophoretic mobility of analytes.

The best results were obtained at $\mathrm{pH} 1.9$, but due to the structural similarities ABZ, FEN, FLZ and MBZ have virtually almost the same electrophoretic mobility. The order of migration was LEV, ABZ, MBZ, FLZ and FBZ, however MBZ and FLZ couldn't be separated on the baseline and the resolution between the four benzimidazole derivatives was relatively low.

In order to improve separation, organic solvents (methanol, acetonitrile) were added to the BGE in concentrations between 5 and 20\%, this led to an increase of the migration times, but the resolution of separation didn't improve. 
Next, we added different $C D$ derivatives in concentration of $5 \mathrm{mM}$ to the buffer electrolyte, positive results were obtained with HP- $\beta-C D$, RAMEB and TRIMEB, but the best results in terms of resolution and migration times were obtained when using RAMEB.

CDs can form complexes with analytes based on their inclusion into the hydrophobic cavity; secondary interactions may include hydrogen bonding or dipole-dipole interactions with the hydroxyl groups on the CDs, or with other polar substituents of the CDs [17].

Due to the different complexation ability of the analytes with the $C D$, the order of migration of the benzimidazole derivatives changed and an increase in the migration time of the analytes was observed. The order of migration in the buffer system containing RAMEB was: LEV, FLZ, ABZ, MBZ and FBZ.

\subsection{Method optimization}

In order to improve the resolution of the separation the influence of several analytical and electrophoretic parameters on the separation (buffer concentration, buffer $\mathrm{pH}, \mathrm{CD}$ concentration, voltage, temperature, injection parameters) were studied. The optimization process was conducted using a "one factor at time" strategy, by changing one parameter at a time, while keeping the other parameters constant.

The effect of $\mathrm{pH}$ of the buffer was investigated over a range between 1.9 and 3.0, an increase of $\mathrm{pH}$ resulted in a small increase in migration times, without affecting considerably the resolution of the separation. At low acid $\mathrm{pH}$ values the effect of EOF is negligible and the analytes will migrate based on their own electrophoretic mobility. Based on the migration times of the analytes, a buffer $\mathrm{pH}$ of 1.9 was chosen.

The effect of BGE concentration was studied by changing the concentration of phosphate from 25 to $100 \mathrm{mM}$ at a constant $\mathrm{pH}$ of 1.9; the use of high concentrations of buffer will result in more Joule heating which will affect peak efficiency and migration time. Based on the migration time and generated current, a $50 \mathrm{mM}$ phosphate buffer was chosen.

The effect of CD concentration (5-20 mM) on the resolution and migration times was studied; high RAMEB concentrations were not preferred due to prolonged analysis time, $5 \mathrm{mM}$ was chosen as a compromise between resolution and migration speed.

The influence of voltage (15-30 kV) over migration time was also investigated; voltage over $25 \mathrm{kV}$ induced an increase in current intensity over $100 \mu \mathrm{A}$; thus $20 \mathrm{kV}$ was selected as optimum.
The influence of capillary temperature $\left(15-30^{\circ} \mathrm{C}\right)$ was evaluated; with the increase of the temperature, migration time decreased; therefore, $25^{\circ} \mathrm{C}$ was chosen as optimum.

The influence of injection time (1-5 s) and injection pressure (25-50 mbar) were studied in order to attain low detection limits without affecting peak shape quality, migration time and resolution; 50 mbar $11 \mathrm{~s}$ was chosen as optimum.

From the above experiments, the optimum conditions for the simultaneous determination of ALB, FBZ, FLZ, MEB and LEV were the following: $50 \mathrm{mM}$ phosphate buffer, $\mathrm{pH}$ 1.9, 5 mM RAMEB buffer additive, applied voltage $20 \mathrm{kV}$, capillary temperature $25^{\circ} \mathrm{C}$, injection parameters $50 \mathrm{mbar} / 1 \mathrm{~s}$, detection wavelength $210 \mathrm{~nm}$. Typical electropherogram obtained under optimized conditions is shown in Fig. 2. Using the optimized conditions, the migration other was the following: LEV, FLZ, ABZ, MBZ and FBZ; all the analytes were separated in approximately $15 \mathrm{~min}$ with resolution (Rs) values over 2.5 and separation factor (a) values over 1.05. Analytical data of the separation are presented in Table 1.

\subsection{Analytical performances}

The intra-day precision of the analytical method was determined by analysing six replicates of a $0.25 \mathrm{mg} / \mathrm{mL}$ standard mixture solution on the same day. Inter-day precision was evaluated with a standard mixture solution containing $0.25 \mathrm{mg} / \mathrm{mL}$ of each analyte, over three consecutive days with six replicates per day. The results were expressed as the percent of relative standard deviation (RSD \%) for migration time, peak area and peak heights. The results presented in Table 2 indicate a good precision of the developed method.

Linearity was studied in a concentration range of 0.01-1 $\mathrm{mg} / \mathrm{mL}$ for each analyte, each calibration curve contained eight different concentrations and was performed in triplicate. Correlation coefficients above 0.99 demonstrate a good linearity of the method. The regression equations and correlation coefficients for the proposed method are shown in Table 3.

Limit of detection (LOD) and limit of quantification (LOQ) were estimated as (standard deviation of regression equation)/(slope of the regression equation) $\times 3.3$ and 10 , respectively, the results are presented in Table 3 . The method possesses adequate sensitivity for the analysis of the analytes in pharmaceutical formulations.

The robustness of the method was evaluated by studying the influence of the variation of several experimental parameters like buffer concentration $(50 \pm 5 \mathrm{mM})$, buffer $\mathrm{pH}(2.2 \pm 0.3), \mathrm{CD}$ concentration $(5 \pm 0.5)$, applied voltage $(20 \pm 2.5 \mathrm{kV})$ and temperature $\left(25 \pm 2{ }^{\circ} \mathrm{C}\right)$, changing only one of these each time, and monitoring variation 


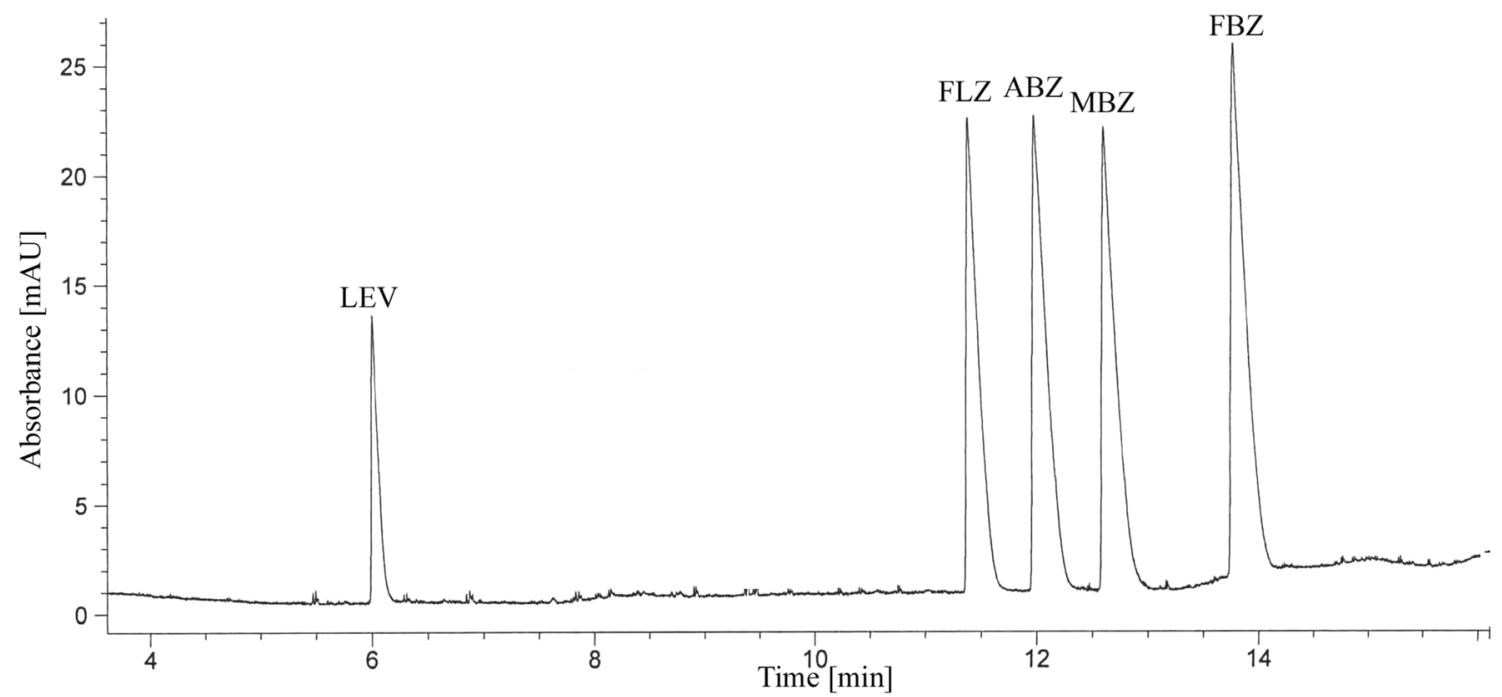

Fig. 2 The separation of the studied anthelmintic drugs under optimized electrophoretic conditions (analytical conditions: $50 \mathrm{mM}$ phosphate buffer, $\mathrm{pH}$ 1.90, 5 mM RAMEB buffer additive,

Table 1 Analytical data for the determination of anthelmintic drugs by CE

\begin{tabular}{lrllll}
\hline Analyte & $\begin{array}{l}\text { Migration } \\
\text { time }( \pm \text { SD) } \\
{[\mathrm{min}](\mathrm{n}=6)}\end{array}$ & Peak symetry & $\begin{array}{l}\text { Number of } \\
\text { theoretical } \\
\text { plates }\end{array}$ & Rs & a \\
\hline LEV & $6.15 \pm 0.01$ & 0.11 & 25,842 & - & - \\
FLZ & $11.65 \pm 0.01$ & 0.14 & 49,467 & 30.33 & 1.90 \\
ABZ & $12.25 \pm 0.02$ & 0.12 & 35,373 & 2.54 & 1.05 \\
MBZ & $12.89 \pm 0.02$ & 0.13 & 41,835 & 2.48 & 1.05 \\
FBZ & $14.08 \pm 0.02$ & 0.14 & 40,748 & 4.47 & 1.09 \\
\hline
\end{tabular}

Table 2 Intra and inter-day precision for the determination of anthelmintic drugs by CE (analyte concentration $0.25 \mathrm{mg} / \mathrm{mL}$ )

\begin{tabular}{llll}
\hline Analyte & $\begin{array}{l}\text { RSD(\%) migration } \\
\text { time }\end{array}$ & $\begin{array}{l}\mathrm{RSD}(\%) \text { peak } \\
\text { area }\end{array}$ & $\begin{array}{l}\mathrm{RSD}(\%) \\
\text { peak } \\
\text { height }\end{array}$ \\
\hline $\begin{array}{l}\text { Intra-day } \\
\text { LEV }\end{array}$ & 0.17 & & \\
FLZ & 0.09 & 0.43 & 0.58 \\
$\mathrm{ABZ}$ & 0.13 & 0.72 & 1.08 \\
$\mathrm{MBZ}$ & 0.15 & 0.36 & 0.68 \\
FBZ & 0.10 & 1.26 & 0.87 \\
Inter-day precision $(\mathrm{n}=6)$ & 1.46 & 0.37 \\
$\mathrm{LEV}$ & 0.49 & & \\
FLZ & 0.76 & 1.18 & 1.26 \\
$\mathrm{ABZ}$ & 0.82 & 2.14 & 1.57 \\
$\mathrm{MBZ}$ & 0.71 & 1.21 & 1.04 \\
FBZ & 0.46 & 2.12 & 1.07 \\
\hline
\end{tabular}

applied voltage $+20 \mathrm{kV}$, temperature $25{ }^{\circ} \mathrm{C}$, injection pressure/ time $50 \mathrm{mbar} / 1 \mathrm{~s}$, sample concentration $0.25 \mathrm{mg} / \mathrm{mL}$, UV detection $210 \mathrm{~nm}$ )

of the migration times. Only small acceptable deviations (RSD $<2 \%$ ) were observed indicating that the robustness of the method was good.

The accuracy of the method was determined by using recovery experiments analysing solutions of known analyte concentrations within the linearity range at three levels $(0.1,0.25,0.5 \mathrm{mg} / \mathrm{mL})$. The mean recoveries were between 98.7 and $102.4 \%$, showing a good accuracy of the method.

The developed method has been successfully applied for the determination of $A B Z$ and MBZ from pharmaceutical preparations. The compounds were found in good agreement with manufacturer's declared values for both samples (Table 4).

The selectivity of the method was tested by comparing the electropherogram of standard solutions and commercial pharmaceutical product solutions containing an equivalent concentration of analytes. No interference occurred from excipients with the drug peaks of the studied analytes during analysis of formulation samples.

\section{Conclusions}

We successfully developed a simple CZE method for the simultaneous determination of $A B Z, F B Z, F L Z, M B Z$ and LEV. Under optimized conditions, using RAMEB as buffer additive we obtained the separation of the five analytes in approximately $15 \mathrm{~min}$. Complexation with CD improved resolution between structurally related compounds, enhancing the selectivity of the method. 
Table 3 Linearity data and LOD/LOQ values for the determination of anthelmintic drugs by $C E$ (concentration range $0.01-1 \mathrm{mg} / \mathrm{mL}, \mathrm{n}=8$ )

\begin{tabular}{lllll}
\hline Analyte & Regression equation & $\begin{array}{l}\text { Correlation coef- } \\
\text { ficient }\end{array}$ & LOD $(\mathrm{mg} / \mathrm{mL})$ & LOQ $(\mathrm{mg} / \mathrm{mL})$ \\
\hline LEV & $y=13.527 x+0.946$ & 0.995 & 0.017 & 0.051 \\
FLZ & $y=11.205 x+1.011$ & 0.991 & 0.012 & 0.037 \\
ABZ & $y=36.277 x+0.282$ & 0.998 & 0.006 & 0.019 \\
MBZ & $y=20.429 x+0.528$ & 0.995 & 0.010 & 0.028 \\
FBZ & $y=45.299 x-0.687$ & 0.998 & 0.004 & 0.012 \\
\hline
\end{tabular}

Table 4 Assay results of ABZ and MBZ in pharmaceutical preparations $(n=3)$

\begin{tabular}{lllll}
\hline Generic name & Analyte & $\begin{array}{l}\text { Label } \\
\text { claim } \\
(\mathrm{mg})\end{array}$ & $\begin{array}{l}\text { Amount found } \\
(\mathrm{mg})\end{array}$ & Recovery (\%) \\
\hline Duador & $\mathrm{ABZ}$ & 200 & $198.5 \pm 1.25$ & 99.25 \\
Vermox & MBZ & 100 & $99.2 \pm 0.87$ & 99.20 \\
\hline
\end{tabular}

The migration order of the benzimidazole analytes can be explained as the analyte which is complexed more strongly by the $C D$ migrates slower; since the hydrodynamic radius of the analyte-CD complex is larger than the radius of the free analyte, the complex will migrate slower. Also, in the migration process molecular weight and analyte size must be taken into consideration, as LEV, the analyte with a significantly lower molecular weight migrates the fastest.

The advantages of our method offer high separation efficiency and minimum use of analytes and solvents, being recommended to be used as quality control protocol in preliminary pharmaceutical analysis. This study highlights the benefits of using universal methods for rapid quantitation of anthelmintic drugs without the need for development of separate and distinct method for each analyte.

\section{Compliance with ethical standards}

Conflict of interest On behalf of all authors, the corresponding author states that there is no conflict of interest.

Human and animal rights This article does not contain any studies with human participants or animals performed by any of the authors.

\section{References}

1. Lo NC, Andrews JR, Bogoch II (2016) Improving helminth treatment access: costs and opportunities. Lancet Infect Dis 16:762-764

2. Grover JK, Vats V, Uppal G, Yadav S (2001) Anthelmintics: a review. Trop Gastroenterol: Off J Dig Dis Found 22:180-189
3. Marsh A, Broderick M, Altria K, Power J, Donegan S, Clark B (2008) Capillary electrophoresis for pharmaceutical analysis. Methods Mol Biol 384:205-245

4. Holzgrabe U, Brinz D, Kopec S, Weber C, Bitar Y (2006) Why not using capillary electrophoresis in drug analysis? Electrophoresis 27:2283-2292

5. Hu XZ, Chen ML, Gao Q, Yu QW, Feng YQ (2012) Determination of benzimidazole residues in animal tissue samples by combination of magnetic solid-phase extraction with capillary zone electrophoresis. Talanta 89:335-341

6. Domínguez-Álvarez J, Mateos-Vivas M, García-Gómez D, Rodríguez-Gonzalo E, Carabias-Martínez R (2013) Capillary electrophoresis coupled to mass spectrometry for the determination of anthelmintic benzimidazoles in eggs using a QuEChERS with preconcentration as sample treatment. J Chromatogr A 1278:166-174

7. Tejada-Casado C, Moreno-Gonzalez D, Lara FJ, Garcia-Campana AM, del Olmo-Iruela M (2017) Determination of benzimidazoles in meat samples by capillary zone electrophoresis tandem mass spectrometry following dispersive liquid-liquid microextraction. J Chromatogr A 1490:212-219

8. Tejada-Casado $C$, Hernández-Mesa $M$, del Olmo-Iruela $M$, García-Campaña AM (2016) Capillary electrochromatography coupled with dispersive liquid-liquid microextraction for the analysis of benzimidazole residues in water samples. Talanta $16: 8-14$

9. Soliman LC, Donkor KK (2014) Micellar electrokinetic chromatography method development for simultaneous determination of thiabendazole, carbendazim, and fuberidazole. $J$ Environ Sci Health, Part B 49:153-158

10. Shen J, Tong J, Jiang H, Rao Q, Li N, Guo L, Ding S (2009) Simultaneous determination of five benzimidazoles in feeds using highperformance capillary electrophoresis. J AOAC Int 92(1009):1015

11. Prochazkova A, Chouki M, Theurillat R, Thormann W (2000) Therapeutic drug monitoring of albendazole: determination of albendazole, albendazole sulfoxide, and albendazole sulfone in human plasma using nonaqueous capillary electrophoresis. Electrophoresis 21:729-736

12. Paias FO, Lanchote VL, Takayanagui OM, Bonato PS (2001) Enantioselective analysis of albendazole sulfoxide in cerebrospinal fluid by capillary electrophoresis. Electrophoresis 22:3263-3269

13. Thormann W, Prost F, Prochazkova A (2002) Capillary electrophoresis with (R)-(-)-N-(3,5-dinitrobenzoyl)-a-phenylglycine as chiral selector for separation of albendazole sulfoxide enantiomers and their analysis in human plasma. J Pharm Bioanal Anal 27:555-567

14. Xu L, Luan F, Hu F, Liu H, Gao Y (2013) Development and validation of a non-aqueous capillary electrophoresis method for simultaneous estimation of mebendazole and levamisole hydrochloride in compound mebendazole tablets. Anal Methods 5:762-766

15. Jerez G, Kayufman G, Prystal M, Schenkeveld S, Donkor KK (2009) Determination of thermodynamic pKa values of 
benzimidazole and benzimidazole derivatives by capillary electrophoresis. J Sep Sci 32:1087-1095

16. Lipka E, Folly-Klan M, Charton J, Vaccher MP, Bonte JP, Vaccher C (2010) Determination of pKa values of benzimidazole derivatives from mobility obtained by capillary electrophoresis. J Pharm Biomed Anal 53:1267-1271

17. Rezanska P, Navratilova K, Rezanska M, Kral V, Sykora D (2014) Application of cyclodextrins in chiral capillary electrophoresis. Electrophoresis 35:2701-2721
Publisher's Note Springer Nature remains neutral with regard to jurisdictional claims in published maps and institutional affiliations. 